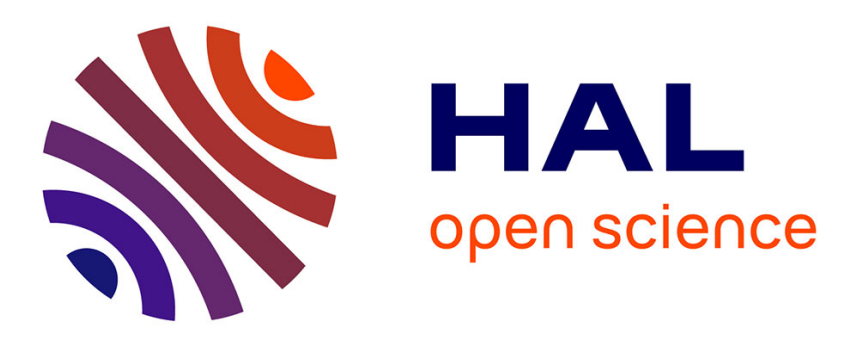

\title{
Scale-free estimation of the average state in large-scale systems
}

\author{
Muhammad Umar B. Niazi, Diego Deplano, Carlos Canudas de Wit, Alain
}

Kibangou

\section{- To cite this version:}

Muhammad Umar B. Niazi, Diego Deplano, Carlos Canudas de Wit, Alain Kibangou. Scale-free estimation of the average state in large-scale systems. IEEE Control Systems Letters, 2019, pp.1-6. 10.1109/LCSYS.2019.2923086 . hal-02158678

\section{HAL Id: hal-02158678 \\ https://hal.science/hal-02158678}

Submitted on 18 Jun 2019

HAL is a multi-disciplinary open access archive for the deposit and dissemination of scientific research documents, whether they are published or not. The documents may come from teaching and research institutions in France or abroad, or from public or private research centers.
L'archive ouverte pluridisciplinaire HAL, est destinée au dépôt et à la diffusion de documents scientifiques de niveau recherche, publiés ou non, émanant des établissements d'enseignement et de recherche français ou étrangers, des laboratoires publics ou privés. 


\title{
Scale-free estimation of the average state in large-scale systems
}

\author{
Muhammad Umar B. Niazi, Diego Deplano, Carlos Canudas-de-Wit, and Alain Y. Kibangou
}

\begin{abstract}
This paper provides a computationally tractable necessary and sufficient condition for the existence of an average state observer for large-scale linear time-invariant (LTI) systems. Two design procedures, each with its own significance, are proposed. When the necessary and sufficient condition is not satisfied, a methodology is devised to obtain an optimal asymptotic estimate of the average state. In particular, the estimation problem is addressed by aggregating the unmeasured states of the original system and obtaining a projected system of reduced dimension. This approach reduces the complexity of the estimation task and yields an observer of dimension one. Moreover, it turns out that the dimension of the system also does not affect the upper bound on the estimation error.
\end{abstract}

Index Terms-Large-scale systems, algebraic/geometric methods, estimation.

\section{INTRODUCTION}

$\mathbf{S}$ TATE estimation for monitoring large-scale systems requires tremendous amounts of computational and sensing resources, which is impractical in most applications, [1]. However, knowledge of some aggregated quantity of the state suffices in several applications.

Processes over physical networks such as traffic [2], epidemic spread [3] and thermal control [4] are examples of large-scale systems. Due to the diffusive nature of these systems, the average state is usually sufficient for monitoring purposes. For instance, estimating the average traffic density in some sector of a traffic network helps to monitor the congestion effectively. In the event of an epidemic, estimating the average proportion of infected people over several towns, which are interconnected through people commuting for work or other purposes, helps to devise the preventive measures for controlling the epidemic spread. For the temperature regulation of a building, the thermistors can only be placed either on the walls or the roof, therefore, estimating the average temperature of the interior of a large corridor is crucial. Other examples include the averaging systems such as opinion networks and wireless sensor networks, [5], where the average state is of paramount importance.

M. U. B. Niazi and C. Canudas-de-Wit are with GIPSA-Lab, CNRS, Grenoble, France (email: muhammad-umar-b.niazi@gipsa-lab.fr; carlos.canudas-dewit@gipsa-lab.fr).

D. Deplano is with DIEE, University of Cagliari, Cagliari, Italy (email: diego.deplano@diee.unica.it).

A. Y. Kibangou is with Univ. Grenoble Alpes, CNRS, Inria, Grenoble INP, GIPSA-Lab, Grenoble, France (email: alain.kibangou@univ-grenoblealpes.fr).

This work is supported by European Research Council (ERC) under the European Union's Horizon 2020 research and innovation programme (ERCAdG no. 694209).
Average state estimation in large-scale network systems has been recently studied in [6], where clustered model reduction [7] is used to reduce the complexity of the estimation task. The aim of [6] is to identify the clusters of network nodes with similar behavior and to estimate the average states of those clusters. For the estimation, an average state observer is designed based on the reduced system, which is obtained by aggregating the clusters. In physical networks, however, the sensors might be already placed and the clusters already specified. Consequently, the clustered model reduction techniques are no more relevant and the estimation task entirely relies on the observer design.

Observer design for estimating a linear functional of the state was first presented in [8]. However, the system is assumed to be observable and the observability index determines the order of the observer, which can be reduced as shown in [9]. Later, in [10]-[12], different methodologies are proposed to obtain a minimum order design of a functional observer. Nonetheless, the conditions to verify the existence of such an observer are not computationally feasible for large-scale systems. This paper, on the other hand, addresses the problem through a projected system which is obtained by projecting the dynamics of the original system to a lower dimensional state space. Such an approach is useful to deal with the complexity of large-scale systems. In particular, the state is partitioned into the measured part, which is the output of the system, and the unmeasured part, whose average we aim to estimate. Note that this approach can be generalized to estimate any linear functional of the state instead of the average.

Average observability is a notion introduced in [13], which studies if it is theoretically possible to reconstruct the average state trajectory of a system from the output measurements. This paper, on the other hand, focuses on the design of an average state observer to obtain an asymptotic estimate of the average state. A necessary and sufficient condition for the existence of such an observer is also provided. When the condition is not satisfied, we provide an observer design methodology to minimize the asymptotic estimation error.

The proposed observer is based on the parameters of the projected system. This approach makes the estimation task 'scale-free,' dual to what is described in [14], since the complexity does not scale with the dimension of the original system. Likewise, it turns out that the upper bound on the estimation error is also invariant to the dimension.

\section{NOTATION}

The real and complex numbers are denoted as $\mathbb{R}$ and $\mathbb{C}$, whereas $\mathbb{R}_{a}$ denotes the reals in the interval $a \subset \mathbb{R}$ and 
$\mathbb{C}_{a}$ denotes the complex plane with real axis restricted to $\mathbb{R}_{a}$. Matrices are denoted by uppercase letters and vectors by boldface lowercase letters. A vector of ones and an identity matrix are denoted as $\mathbf{1}_{n}$ and $I_{n}$, respectively, whereas $0_{n \times m}$ and $\mathbf{0}_{n}$ denote a matrix and a vector of zeros, respectively, where subscripts (sometimes omitted for brevity) indicate their dimensions. For a matrix $A \in \mathbb{R}^{n \times m}$, we denote its transpose and pseudo-inverse by $A^{T}$ and $A^{+}$, respectively; and, when $n=m, \operatorname{eig}(A)$ denotes the set of its eigenvalues. A diagonal matrix is written as $\operatorname{diag}\left[a_{1}, \cdots, a_{n}\right]$ with elements $a_{1}, \cdots, a_{n}$ at its diagonal. The absolute value of a scalar is denoted by $|\cdot|$ and a generic p-norm for vectors by $\|\cdot\|$. With each vector norm $\|\cdot\|$ on $\mathbb{R}^{n}$, we associate a matrix norm $\|\cdot\| \|$ induced by $\|\cdot\|$ on $\mathbb{R}^{n \times n}$.

\section{PROBLEM DEFINITION}

Linear time-invariant (LTI) systems of the form

$$
\begin{aligned}
& \dot{\mathbf{x}}(t)=A \mathbf{x}(t)+B \mathbf{u}(t) \\
& \mathbf{y}(t)=C \mathbf{x}(t)
\end{aligned}
$$

are considered, where $\mathbf{x}(t) \in \mathbb{R}^{n}, \mathbf{u}(t) \in \mathbb{R}^{p}$, and $\mathbf{y}(t) \in \mathbb{R}^{m}$ are the state, input, and output vectors, respectively, and $A \in \mathbb{R}^{n \times n}, B \in \mathbb{R}^{n \times p}, C \in \mathbb{R}^{m \times n}$ are constant matrices. To emphasize the scope of the paper and avoid trivial cases, we suppose that $m<n / 2$ and the pair $(C, A)$ is neither observable nor detectable.

Without loss of generality, we assume the partition of the state vector $\mathbf{x}(t)=\left[\begin{array}{ll}\mathbf{x}_{1}^{T}(t) & \mathbf{x}_{2}^{T}(t)\end{array}\right]^{T}$, where $\mathbf{x}_{1}(t) \in \mathbb{R}^{k}$ and $\mathbf{x}_{2}(t)=\mathbf{y}(t) \in \mathbb{R}^{m}$ with $n=k+m$. Thus, the system matrices are partitioned as

$$
\begin{aligned}
& A=\left[\begin{array}{ll}
A_{11} & A_{12} \\
A_{21} & A_{22}
\end{array}\right], \quad B=\left[\begin{array}{l}
B_{1} \\
B_{2}
\end{array}\right], \\
& C=\left[\begin{array}{ll}
0_{m \times k} & I_{m}
\end{array}\right] ;
\end{aligned}
$$

where the dimensions of the matrix blocks are in accordance with the state partition.

Referring to $\mathbf{x}_{1}(t)$ and $\mathbf{x}_{2}(t)$ as unmeasured and measured states, respectively, we aim to estimate the average of the unmeasured state, i.e. $z_{1}(t)=\frac{1}{k} \mathbf{1}_{k}^{T} \mathbf{x}_{1}(t)$. With the estimate of $z_{1}(t)$ and the measurements $\mathbf{x}_{2}(t)$, one can obtain an estimate of the average of the whole state $\mathbf{x}(t)$.

We obtain a lower-order projected system by aggregating the unmeasured state $\mathbf{x}_{1}(t)$ as

$$
\begin{aligned}
\dot{\mathbf{z}}(t) & =E \mathbf{z}(t)+F \boldsymbol{\sigma}(t)+G \mathbf{u}(t) \\
\mathbf{y}(t) & =H \mathbf{z}(t)
\end{aligned}
$$

where the state vector $\mathbf{z}(t)=\left[\begin{array}{ll}z_{1}(t) & \mathbf{x}_{2}^{T}(t)\end{array}\right]^{T} \in \mathbb{R}^{m+1}$ and the matrices are given as

$$
\begin{array}{llrl}
E & =\left[\begin{array}{rr}
\frac{1}{k} \mathbf{1}^{T} A_{11} \mathbf{1} & \frac{1}{k} \mathbf{1}^{T} A_{12} \\
A_{21} \mathbf{1} & A_{22}
\end{array}\right], & F=\left[\begin{array}{r}
\frac{1}{k} \mathbf{1}^{T} A_{11} \\
A_{21}
\end{array}\right] \\
H=\left[\begin{array}{lll}
\mathbf{0}_{m} & I_{m}
\end{array}\right], & G=\left[\begin{array}{r}
\frac{1}{k} \mathbf{1}^{T} B_{1} \\
B_{2}
\end{array}\right] .
\end{array}
$$

The average deviation vector $\boldsymbol{\sigma}(t) \in \mathbb{R}^{k}$ is given by $\boldsymbol{\sigma}(t):=$ $\left(I-\frac{1}{k} \mathbf{1}_{k} \mathbf{1}_{k}^{T}\right) \mathbf{x}_{1}(t)$, where we note that

$$
\mathbf{1}_{k}^{T} \boldsymbol{\sigma}(t)=0
$$

for all $t \in \mathbb{R}_{>0}$.

The goal is to devise an observer whose estimate $\hat{z}_{1}(t)$ converges to the true value $z_{1}(t)$ asymptotically as $t \rightarrow \infty$. This necessarily involves canceling the effect of $\boldsymbol{\sigma}(t)$ from $\boldsymbol{\Sigma}_{\boldsymbol{\Sigma}}^{\circ}$. Thus the problem considered here is similar to the problem of observer design for systems with unknown inputs, [15], [16], where $\boldsymbol{\sigma}(t)$ is assumed to be completely arbitrary. However, in our case, $\boldsymbol{\sigma}(t)$ is not arbitrary but satisfies (3). Hence, it is quite intuitive that the possibility to cancel the effect of $\boldsymbol{\sigma}(t)$ is necessary for the existence of an average state observer. This consideration directs the study in two directions: 1) Find a necessary and sufficient condition under which an average state observer exists. 2) If the average state observer doesn't exist, devise a methodology to obtain an optimal estimate $\hat{z}_{1}(t)$ as $t \rightarrow \infty$.

\section{Asymptotic EStimation of the AVERAGE STATE}

The following form of the observer is considered

$$
\begin{aligned}
& \dot{\mathbf{w}}(t)=M \mathbf{w}(t)+K \mathbf{y}(t)+N G \mathbf{u}(t), \\
& \hat{\mathbf{z}}(t)=\mathbf{w}(t)+L \mathbf{y}(t)
\end{aligned}
$$

where $\mathbf{w}(t), \hat{\mathbf{z}}(t) \in \mathbb{R}^{m+1}$ are the state and output of $\widehat{\boldsymbol{\Omega}}$, respectively, and $M, K, N, L$ are matrices of appropriate dimensions. Let the estimation error be $\tilde{\mathbf{z}}(t):=\mathbf{z}(t)-\hat{\mathbf{z}}(t)$, which satisfies

$$
\dot{\tilde{\mathbf{z}}}(t)=M \tilde{\mathbf{z}}(t)+N F \boldsymbol{\sigma}(t),
$$

where

$$
\begin{array}{ll}
N=I-L H, & M=N E-K_{1} H, \\
K_{2}=M L, & K=K_{1}+K_{2} .
\end{array}
$$

If $\|\tilde{\mathbf{z}}(t)\| \rightarrow 0$ as $t \rightarrow \infty$, then the observer $\widehat{\Omega}$ exists and estimates $\mathbf{z}(t)$ asymptotically.

In what follows, we abide by the assumption that the pair $(H, E)$ is observable, i.e. $\operatorname{rank} \mathcal{O}_{H, E}=m+1$, where $H, E$ are given in (2) and

$$
\mathcal{O}_{H, E}=\left[\begin{array}{llll}
H^{T} & (H E)^{T} & \cdots & \left(H E^{m}\right)^{T}
\end{array}\right]^{T} .
$$

Lemma IV.1. The pair $(H, E)$ is observable if and only if $A_{21} \mathbf{1}_{k} \neq \mathbf{0}_{m}$, where $H, E$ are given in (2) and $A_{21}$ in (1).

The proof of this Lemma is provided in [13]. The condition $A_{21} \mathbf{1} \neq 0$ implies that the average state affects the dynamics of at least one measured state. This condition is necessary for the observer $\widehat{\Omega}$ to asymptotically estimate the average state from the output $\mathbf{y}(t)$ and the input $\mathbf{u}(t)$.

\section{A. Necessary and sufficient condition}

We provide a necessary and sufficient condition that is easy to check computationally since it doesn't require, unlike [12], to construct several observability matrices, which is computationally not feasible for large-scale systems.

Theorem IV.1. Given $\boldsymbol{\Sigma}$, consider $\stackrel{\circ}{\boldsymbol{\Sigma}}$ and $\widehat{\boldsymbol{\Omega}}$. Assume that $(H, E)$ is an observable pair. Then, the estimation error $\tilde{\mathbf{z}}(t):=\mathbf{z}(t)-\hat{\mathbf{z}}(t)$ converges to 0 as $t \rightarrow \infty$ at an arbitrary rate if and only if

$$
\operatorname{rank}\left[\begin{array}{c}
\mathbf{1}_{k}^{T} \\
\mathbf{1}_{k}^{T} A_{11} \\
A_{21}
\end{array}\right]=\operatorname{rank} A_{21},
$$


where $\mathbf{1}_{k}^{T} A_{11} \in \mathbb{R}^{1 \times k}$ and $A_{21} \in \mathbb{R}^{m \times k}$ are given in (2).

Proof: Recall the error dynamics in (4) and the property (3). Then, since $\boldsymbol{\sigma}(t) \in \mathbb{R}^{k}$, we note that $N F \boldsymbol{\sigma}(t)=0$ for all $t \in \mathbb{R}_{\geq 0}$ if and only if $N F=\mathbf{v} \mathbf{1}^{T}$ for some $\mathbf{v} \in \mathbb{R}^{m+1}$, where $F$ is given in (2) and $N$ in (5). The equation $N F=\mathbf{v} \mathbf{1}^{T}$ with $N F=(I-L H) F$ can be written as

$$
\left[\begin{array}{cc}
1 & -\ell_{1}^{T} \\
\mathbf{0}_{m} & I_{m}-L_{2}
\end{array}\right]\left[\begin{array}{c}
\frac{1}{k} \mathbf{1}^{T} A_{11} \\
A_{21}
\end{array}\right]=\left[\begin{array}{c}
v_{1} \mathbf{1}^{T} \\
\mathbf{v}_{2} \mathbf{1}^{T}
\end{array}\right],
$$

where $L=\left[\begin{array}{c}\ell_{1}^{T} \\ L_{2}\end{array}\right] \in \mathbb{R}^{(m+1) \times m}$ and $\mathbf{v}=\left[\begin{array}{c}v_{1} \\ \mathbf{v}_{2}\end{array}\right] \in \mathbb{R}^{m+1}$. For some arbitrary $\mathbf{v}$, we find $L$ such that (8) is satisfied. Hence, to satisfy the upper part of (8), we must have

$$
\operatorname{rank}\left[\begin{array}{c}
\frac{1}{k} \mathbf{1}^{T} A_{11}-v_{1} \mathbf{1}^{T} \\
A_{21}
\end{array}\right]=\operatorname{rank} A_{21},
$$

for some $v_{1} \in \mathbb{R}$. The lower part of (8) is satisfied either if $L_{2} \neq I_{m}$ and

$$
\operatorname{rank}\left[\begin{array}{c}
\mathbf{1}^{T} \\
A_{21}
\end{array}\right]=\operatorname{rank} A_{21},
$$

or if $L_{2}=I_{m}$. We prove the necessity and sufficiency of the theorem as follows.

Sufficiency: If $L_{2} \neq I_{m}$, then $N=I-L H$ is such that the pair $(H, N E)$ is observable (it can be verified by checking the rank of $\mathcal{O}_{H, N E}$ by replacing $E$ with $N E$ in (6)). Therefore, $\operatorname{eig}(M) \subset \mathbb{C}_{<0}$ can be assigned arbitrarily by the algorithm presented in [17]. Thus $\|\tilde{\mathbf{z}}(t)\| \rightarrow 0$ as $t \rightarrow \infty$ at an arbitrary rate determined by $\operatorname{eig}(M) \subset \mathbb{C}_{<0}$. To satisfy (8) for $L_{2} \neq I_{m}$, the condition (10) must hold. Since (7) implies (9) and (10), the sufficiency of (7) is proved.

Necessity: If $L_{2}=I_{m}$, then $N=I-L H$ is such that $(H, N E)$ is not an observable pair. However, if we choose $K=\left[\begin{array}{ll}\mathbf{k}_{11} & K_{12}^{T}\end{array}\right]^{T}$ such that $\mathbf{k}_{11}^{T}=\frac{1}{k} \mathbf{1}^{T} A_{12}-\boldsymbol{\ell}_{1}^{T} A_{22}$ and $K_{12}=-\operatorname{diag}\left[\lambda_{2}, \cdots, \lambda_{m+1}\right]$, then we have

$$
M=\operatorname{diag}\left[\lambda_{1}\left(v_{1}\right), \lambda_{2}, \cdots, \lambda_{m+1}\right],
$$

where $\lambda_{1}\left(v_{1}\right)=\frac{1}{k} \mathbf{1}^{T} A_{11}\left(I-A_{21}^{+} A_{21}\right) \mathbf{1}+v_{1} \mathbf{1}^{T} A_{21}^{+} A_{21} \mathbf{1}$ and $\lambda_{i} \in \mathbb{R}_{<0}$ for $i=2, \cdots, m+1$. For $M$ to be a Hurwitz matrix, it is necessary that

$$
v_{1}<\frac{\mathbf{1}^{T} A_{11}\left(A_{21}^{+} A_{21}-I\right) \mathbf{1}}{k \mathbf{1}^{T} A_{21}^{+} A_{21} \mathbf{1}} .
$$

Furthermore, to have $\lambda_{1}\left(v_{1}\right) \in \mathbb{R}_{<0}$ arbitrary, i.e. $\|\tilde{\mathbf{z}}(t)\| \rightarrow 0$ as $t \rightarrow \infty$ at an arbitrary rate determined by $\operatorname{eig}(M) \subset \mathbb{R}_{<0}$, it must hold that (9) is satisfied for all $v_{1} \in \mathbb{R}$ satisfying (12). In other words, the rows of $A_{21}$ must span the plane formed by $\operatorname{span}\left\{\mathbf{1}^{T} A_{11}, \mathbf{1}^{T}\right\}$ excluding the line $\operatorname{span}\left\{\mathbf{1}^{T}\right\}$. That is, for general $\Sigma$, (9) must hold for all $v_{1} \in \mathbb{R}$, which proves the necessity of (7).

\section{B. Design procedures for average state observer}

As a consequence of the proof of Theorem IV.1, we develop two design procedures for $\Omega$. The first design provides $L$ such that the pair $(H, N E)$ is observable, where $N=I-L H$. Hence, $\operatorname{eig}(M) \subset \mathbb{C}_{<0}$ can be arbitrarily assigned by finding the matrix $K_{1}$ by the pole-placement algorithm [17]. The second provides $L$ and $K$ such that $M$ is a diagonal matrix with entries in $\mathbb{R}_{<0}$.

Proposition IV.2 (Design 1). Let the system $\Sigma$ be such that (7) is satisfied. Consider the observer $\widehat{\Omega}$ such that

$$
\begin{aligned}
L & =\left[\begin{array}{c}
\frac{1}{k} \mathbf{1}^{T} A_{11}-v_{1} \mathbf{1}^{T} \\
A_{21}-\mathbf{v}_{2} \mathbf{1}^{T}
\end{array}\right] A_{21}^{+}, \\
K_{1} & =\operatorname{place}\left(N E, H,\left[\lambda_{1}, \ldots, \lambda_{m+1}\right]\right),
\end{aligned}
$$

where $\lambda_{i} \in \mathbb{C}_{<0}$, for $i=1, \cdots, m+1$, are the desired eigenvalues of $M$, "place" is the pole-placement algorithm provided in [17], $v_{1} \in \mathbb{R}$ and $\mathbf{v}_{2} \in \mathbb{R}^{m} \backslash\left\{\mathbf{0}_{m}\right\}$ are arbitrary, and the matrices $M, N, K$ are chosen according to (5). Then, the estimation error $\tilde{\mathbf{z}}(t)$ converges to 0 as $t \rightarrow \infty$ at a rate determined by $\operatorname{eig}(M)=\left\{\lambda_{1}, \cdots, \lambda_{m+1}\right\}$.

Proof: Since (7) is satisfied, $L$ in (13) is the solution satisfying (8) for some arbitrary values of $v_{1} \in \mathbb{R}$ and $\mathbf{v}_{2} \in \mathbb{R}^{m}$, see [18]. Therefore, $N F \boldsymbol{\sigma}(t)=0$ for all $t \in \mathbb{R}_{\geq 0}$. Moreover, the pair $(H, N E)$ is observable (refer to the sufficiency part of the proof of Theorem IV.1). Therefore, $K_{1}$ obtained by the pole placement algorithm [17] gives $M=N E-K_{1} H$ with $\operatorname{eig}(M)=\left\{\lambda_{1}, \cdots, \lambda_{m+1}\right\}$, where $\lambda_{i} \in \mathbb{C}_{<0}$.

Proposition IV.3 (Design 2). Let the system $\boldsymbol{\Sigma}$ be such that (7) is satisfied. Consider the observer $\widehat{\Omega}$ such that

$$
\begin{aligned}
& L=\left[\begin{array}{l}
\ell_{1}^{T} \\
L_{2}
\end{array}\right]=\left[\begin{array}{c}
\left(\frac{1}{k} \mathbf{1}^{T} A_{11}-v_{1} \mathbf{1}^{T}\right) A_{21}^{+} \\
I_{m}
\end{array}\right], \\
& K_{1}=\left[\begin{array}{c}
\mathbf{k}_{11}^{T} \\
K_{12}
\end{array}\right]=\left[\begin{array}{c}
\frac{1}{k} \mathbf{1}^{T} A_{12}-\boldsymbol{\ell}_{1}^{T} A_{22} \\
-\operatorname{diag}\left[\lambda_{2}, \cdots, \lambda_{m+1}\right]
\end{array}\right],
\end{aligned}
$$

where $\lambda_{i} \in \mathbb{R}_{<0}$, for $i=2, \cdots, m+1$, and $v_{1} \in \mathbb{R}$ such that (12) is satisfied, and the matrices $M, N, K$ chosen according to (5). Then, the estimation error $\tilde{\mathbf{z}}(t)$ converges to 0 as $t \rightarrow \infty$ at a rate determined by $\operatorname{eig}(M)=\left\{\lambda_{1}\left(v_{1}\right), \cdots, \lambda_{m+1}\right\}$, where $M$ is given in (11) and

$$
\lambda_{1}\left(v_{1}\right)=\frac{1}{k} \mathbf{1}^{T} A_{11}\left(I-A_{21}^{+} A_{21}\right) \mathbf{1}+v_{1} \mathbf{1}^{T} A_{21}^{+} A_{21} \mathbf{1} .
$$

Proof: The choice of $L$ is such that $N F \boldsymbol{\sigma}(t)=0$ for all $t \in \mathbb{R}_{\geq 0}$. Moreover, the choice of matrices in (14) yields a diagonal $M$, as in (11), where $v_{1}$ is chosen such that it satisfies (12). Therefore, $\operatorname{eig}(M) \subset \mathbb{R}_{<0}$ are assigned arbitrarily and $\|\tilde{\mathbf{z}}(t)\| \rightarrow 0$ as $t \rightarrow \infty$.

In design 2 , the eigenvalues can only be chosen in $\mathbb{R}_{<0}$ and not in $\mathbb{C}_{<0}$. However, this design yields a reduced-order average observer of dimension equal to 1 .

Proposition IV.4 (Reduced-order average observer). Consider the observer $\widehat{\Omega}$. Then, the choice of design matrices in (14) yields a reduced order average observer

$$
\begin{aligned}
& \dot{w}_{1}(t)=\lambda_{1}\left(v_{1}\right) w_{1}(t)+\mathbf{k}_{11}^{T} \mathbf{y}(t)+\mathbf{g}^{T} \mathbf{u}(t) \\
& \hat{z}_{1}(t)=w_{1}(t)+\ell_{1}^{T} \mathbf{y}(t)
\end{aligned}
$$

such that $z_{1}(t)-\hat{z}_{1}(t) \rightarrow 0$ as $t \rightarrow \infty$, where $w_{1}(t) \in \mathbb{R}, \ell_{1}^{T}$ and $\mathbf{k}_{11}$ given in (14), $\lambda_{1}\left(v_{1}\right)$ given in (15), and

$$
\mathbf{g}^{T}=\left(\frac{1}{k} \mathbf{1}^{T} B_{1}-\ell_{1}^{T} B_{2}\right) G
$$

with $B_{1} \in \mathbb{R}^{k \times p}$ and $B_{2} \in \mathbb{R}^{m \times p}$ given in (1). 
Proof: We can obtain $\widehat{\boldsymbol{\Omega}}_{1}$ from $\widehat{\boldsymbol{\Omega}}$ since all states in $\widehat{\boldsymbol{\Omega}}$ under (14) are decoupled and are stable.

The significance of both design procedures are summarized as follows. In design 1 , eig $(M)$ can be arbitrarily assigned in $\mathbb{C}_{<0}$, which gives an extra control over the estimation performance in the transient phase. Design 2, on the other hand, enables us to obtain a reduced-order average observer $\widehat{\Omega}_{1}$ of dimension equal to 1 , which makes the estimation problem scale-free.

\section{Approximate estimation of the aVerage state}

If a system $\boldsymbol{\Sigma}$ doesn't satisfy the necessary and sufficient condition in Theorem IV.1, the estimation error doesn't converge to zero. In this section, we devise a methodology to minimize $\lim \sup _{t \rightarrow \infty}\|\tilde{\mathbf{z}}(t)\|$.

\section{A. Boundedness of the estimation error}

We prove under suitable assumptions that the estimation error remains bounded, i.e. $\lim \sup _{t \rightarrow \infty}\|\tilde{\mathbf{z}}(t)\| \leq \delta$ where $0 \leq \delta<\infty$ is an upper bound. The result in this subsection is a gateway to error minimization considered in the next subsection.

Theorem V.1. Consider $\boldsymbol{\Sigma}, \stackrel{\circ}{\Sigma}$, and $\widehat{\boldsymbol{\Omega}}$ such that $\tilde{\mathbf{z}}(t)$ satisfies (4). Assume one of the following:

(i) $\operatorname{eig}(A) \subset \mathbb{C}_{\leq 0}$ and $\int_{0}^{\infty}\|\mathbf{u}(t)\| d t<\infty$.

(ii) $\operatorname{eig}(A) \subset \mathbb{C}_{<0}$ and $\|\mathbf{u}(t)\|<\infty$ for all $t \in \mathbb{R}_{\geq 0}$.

Then, $\lim \sup _{t \rightarrow \infty}\|\tilde{\mathbf{z}}(t)\|$ is bounded.

Proof: Consider (4), where the error trajectory satisfies $\|\tilde{\mathbf{z}}(t)\| \leq\left\|e^{M t} \tilde{\mathbf{z}}(0)\right\|+\left\|\int_{0}^{t} e^{M(t-\tau)} N F \boldsymbol{\sigma}(\tau) d \tau\right\|$. Note that $M$ can be chosen to be Hurwitz (i.e. all eigenvalues have negative real parts). Thus, $\lim _{t \rightarrow \infty}|| e^{M t} \mid \|=0$ and

$$
\begin{aligned}
\limsup _{t \rightarrow \infty}\|\tilde{\mathbf{z}}(t)\| & \leq \limsup _{t \rightarrow \infty}\left\|\int_{0}^{t} e^{M(t-\tau)} N F \boldsymbol{\sigma}(\tau)\right\| d \tau \\
& \leq \limsup _{t \rightarrow \infty} \int_{0}^{t}\left\|e^{M(t-\tau)} N F \boldsymbol{\sigma}(\tau)\right\| d \tau \\
& \leq \limsup _{t \rightarrow \infty} \int_{0}^{t}\left\|e^{M(t-\tau)}\right\|\|N F \boldsymbol{\sigma}(\tau)\| d \tau .
\end{aligned}
$$

Since the two functions inside the integral are positive by definition of the norm, it holds (see Section 23 of [19])

$$
\begin{aligned}
\limsup _{t \rightarrow \infty}\|\tilde{\mathbf{z}}(t)\| & \leq\|N F \boldsymbol{\sigma}\|_{\infty} \lim _{t \rightarrow \infty} \int_{0}^{t}\left\|e^{M(t-\tau)}\right\| \| d \tau \\
& \leq \frac{\left\|V^{-1}\right\| \mid\|V\|\|N F J\|}{\lambda^{*}}\left\|\mathbf{x}_{1}\right\|_{\infty}
\end{aligned}
$$

where $J=I_{k}-\frac{1}{k} \mathbf{1}_{k} \mathbf{1}_{k}^{T}, \lambda^{*}=\min _{\lambda \in \operatorname{eig}(M)}|\operatorname{Re}\{\lambda\}|>0$, $\left\|\mathbf{x}_{1}\right\|_{\infty}=\sup \left\{\left\|\mathbf{x}_{1}(t)\right\|: t \in \mathbb{R}_{\geq 0}\right\}$, and $V$ is the matrix of eigenvectors in eigenvalue decomposition of $M$. The inequality (16) is obtained since

$$
\begin{aligned}
\lim _{t \rightarrow \infty} \int_{0}^{t}\|\| e^{M(t-\tau)}\|\| d \tau & =\int_{0}^{\infty}\|\mid\| e^{M \tau}\|\| d \tau \\
& \leq \frac{\left\|V^{-1}\right\||||| V\|\|}{\lambda^{*}}
\end{aligned}
$$

Note that, if either of the assumptions (i) and (ii) hold, then we have $\left\|\mathbf{x}_{1}(t)\right\|<\infty$ for all $t \in \mathbb{R}_{\geq 0}$, which completes the proof.

The bound on the error (16) depends on the condition number of $V$, which is defined as $\operatorname{cond}(V):=\left|\left\|V^{-1}|\|\mid\| V \|\right.\right.$. An upper bound on the condition number of matrices is widely studied, see [20], however, to our knowledge, there is no upper bound on the condition number of eigenvector matrix $V$. Therefore, in general, to minimize $\|N F J\|$ by a suitable choice of $N$ doesn't ensure the minimization of the bound (16) in the case of design 1 (Proposition IV.2). However, in design 2 (Proposition IV.3), the matrix $M$ is diagonal, therefore $V=I$ and $\operatorname{cond}(V)=1$.

\section{B. Minimization of the asymptotic estimation error}

We devise a methodology based on design 2 (Proposition IV.3) to obtain an optimal average state estimate as $t \rightarrow \infty$. Since $M$ is diagonal, (16) is given by

$$
\limsup _{t \rightarrow \infty}\|\tilde{\mathbf{z}}(t)\| \leq \frac{\left\|N\left(v_{1}\right) F J\right\|}{\lambda^{*}}\left\|\mathbf{x}_{1}\right\|_{\infty} .
$$

The eigenvalues $\lambda_{2}, \cdots, \lambda_{m+1}$ of $M$ can be chosen freely, therefore we assume that $\lambda^{*}=\left|\lambda_{1}\left(v_{1}\right)\right|$, where $\lambda_{1}\left(v_{1}\right)$ is given in (15).

Proposition V.2. Consider $\widehat{\Omega}$ with design (14). Assume the conditions of Theorem V.1 hold such that (17) is bounded. Then, the bound (17) is minimized by $v_{1} \in \mathbb{R}$, which is the solution to

$$
\begin{array}{ll}
\min _{v_{1} \in \mathbb{R}} & \frac{\left\|v_{1} \mathbf{p}^{T}-\mathbf{q}^{T}\right\|}{\left|v_{1} \alpha-\beta\right|} \\
\text { subject to } & v_{1} \alpha-\beta<0,
\end{array}
$$

where $\mathbf{p}^{T}=\mathbf{1}^{T}\left(A_{21}^{+} A_{21}-I\right), \mathbf{q}^{T}=\frac{1}{k} \mathbf{1}^{T} A_{11}\left(A_{21}^{+} A_{21}-I\right)$, $\alpha=\mathbf{p}^{T} \mathbf{1}+k, \beta=\mathbf{q}^{T} \mathbf{1}$, and $\lambda^{*}=\left|\alpha v_{1}-\beta\right|$. Here, we assume that $\alpha \neq 0$ and $\beta \neq 0$.

Proof: Consider (17). If (7) doesn't hold, then $\|N F J\| \neq 0$, where $F, N$, and $J$ are given in (2), (5), and (16), respectively. Note that $N F J=0$ if and only if $N F=\mathbf{v 1} 1^{T}$ since rank $J=$ $k-1$, where $\mathbf{v}=\left[\begin{array}{ll}v_{1} & \mathbf{v}_{2}^{T}\end{array}\right]^{T} \in \mathbb{R}^{m+1}$. Thus minimizing $\|N F J\|$ is equivalent to minimizing $\left\|N F-\mathbf{v} \mathbf{1}^{T}\right\|$, where $\mathbf{v} \in \mathbb{R}^{m+1}$ is a free parameter and

$$
N F-\mathbf{v} \mathbf{1}^{T}=\left[\begin{array}{c}
\frac{1}{k} \mathbf{1}^{T} A_{11}-\ell_{1}^{T} A_{21}-v_{1} \mathbf{1}^{T} \\
\left(I_{m}-L_{2}\right) A_{21}-\mathbf{v}_{2} \mathbf{1}^{T}
\end{array}\right] .
$$

For a given $\mathbf{v}$, the solution to this minimization problem is given by the method of least squares of the corresponding linear equations, see [18], which gives an analytic solution of $L$ in terms of $\mathbf{v}$ as given in (13). However, notice that $\mathbf{v}_{2}=\mathbf{0}_{m}$ is necessary for a minimizing solution, which gives a matrix $L$ as given in (14) in terms of $v_{1} \in \mathbb{R}$. Therefore, if $\lambda^{*}=\left|\alpha v_{1}-\beta\right|$, the bound on the steady state estimation error (16) is minimized by minimizing the following

$$
\min _{L\left(v_{1}\right)} \frac{\|N F J\|}{\lambda^{*}}=\min _{v_{1}} \frac{\left\|\left(\frac{1}{k} \mathbf{1}^{T} A_{11}-v_{1} \mathbf{1}^{T}\right)\left(I-A_{21}^{+} A_{21}\right)\right\|}{\left|\alpha v_{1}-\beta\right|},
$$

where $\alpha, \beta$ are given in (18). 


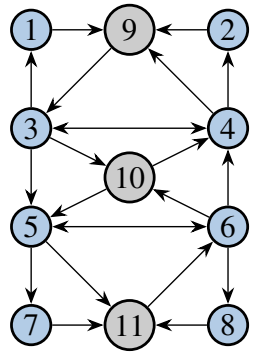

(a) Example 1

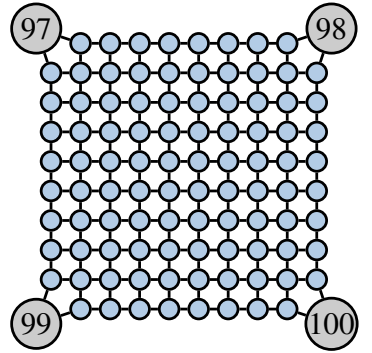

(b) Example 2
Fig. 1: Graph structures for simulation examples

The optimization problem (18) can be solved by an appropriate optimization technique. However, if the norm function in (18) is chosen to be differentiable, one obtains an analytic solution for optimal $v_{1}^{*}$.

Corollary V.2.1. Consider the minimization problem (18) with a Euclidean norm, then the optimal solution is given by

$$
v_{1}^{*}= \begin{cases}\frac{\alpha \mathbf{q}^{T} \mathbf{q}-\beta \mathbf{p}^{T} \mathbf{q}}{\beta \mathbf{p}^{T} \mathbf{p}-\alpha \mathbf{p}^{T} \mathbf{q}}, & \text { if } \frac{\alpha^{2}}{\beta^{2}}<\frac{\mathbf{p}^{T} \mathbf{p}}{\mathbf{q}^{T} \mathbf{q}}, \\ \frac{\beta^{2} \mathbf{p}^{T} \mathbf{p}-\alpha \beta \mathbf{p}^{T} \mathbf{q}}{\alpha^{2} \mathbf{p}^{T} \mathbf{q}-\alpha \beta \mathbf{p}^{T} \mathbf{p}}, & \text { otherwise; }\end{cases}
$$

where $\alpha, \beta, \mathbf{p}, \mathbf{q}$ are given in (18). Here, we assume that $\beta \mathbf{p}^{T} \mathbf{p} \neq \alpha \mathbf{p}^{T} \mathbf{q}$ or $\alpha \mathbf{p}^{T} \mathbf{q} \neq \beta \mathbf{p}^{T} \mathbf{p}$.

Remark V.1. The error bound (17) doesn't increase with the dimension of the system. To illustrate, note that $\left\|\mathbf{x}_{1}\right\|_{\infty}$ depends on the system dynamics and $N F=\left[\begin{array}{c}\mathbf{r}^{T} \\ 0_{m \times k}\end{array}\right]$, where $\mathbf{r}^{T}=\frac{1}{k} \mathbf{1}^{T} A_{11}\left(I-A_{21}^{+} A_{21}\right)+v_{1} \mathbf{1}^{T} A_{21}^{+} A_{21}$. Then, $\lambda_{1}=\mathbf{r}^{T} \mathbf{1}$, from (15), and we have, from (17),

$$
\frac{\|N F J\|}{\left|\lambda_{1}\right|}=\frac{\left\|\mathbf{r}^{T} J\right\|}{\left|\mathbf{r}^{T} \mathbf{1}\right|} \leq \frac{\|\mathbf{r}\|}{\left|\mathbf{r}^{T} \mathbf{1}\right|}+\frac{1}{\sqrt{k}} .
$$

If $\mathbf{r}$ is either nonnegative or nonpositive element-wise, then $\frac{\|\mathbf{r}\|}{\left|\mathbf{r}^{T} \mathbf{1}\right|} \leq 1$ since $\left|\mathbf{r}^{T} \mathbf{1}\right|=\|\mathbf{r}\|_{1}$.

\section{SimUlation EXAMPLES}

In this section, we present examples to illustrate the design procedures provided in the preceding sections. For large-scale systems, we represent the state matrix $A$ by a graph, where the nodes represent the states of the system and the edges represent the positive off-diagonal entries of $A$.

Example VI.1. A linear compartmental system [21] with a structure as shown in Fig. 1(a) is considered, where blue nodes represent the unmeasured states and grey nodes the measured states. The state at each node $i$ satisfies $\dot{x}_{i}(t)=$ $\sum_{j=1}^{n} a_{i j} x_{j}(t)-\sum_{h=1}^{n} a_{h i} x_{i}(t)$, where $a_{i j}=[A]_{i j}$ with $[A]_{i j}=1$ if there is edge $(i, j)$, for $i \neq j$, and 0 otherwise. By constructing the matrix $A$, it can be verified that (7) is satisfied. We generate $\mathbf{x}(0)$ uniformly random in $\mathbb{R}_{(-2,2)}^{n}$ with $n=11$. The output matrix $C=\left[\begin{array}{ll}0_{3 \times 8} & I_{3}\end{array}\right]$ and the input matrix $B=C^{T}$ with $\mathbf{u}(t)=10\left[\begin{array}{lll}\sin t & \sin 10 t & \sin 20 t\end{array}\right]^{T}$.

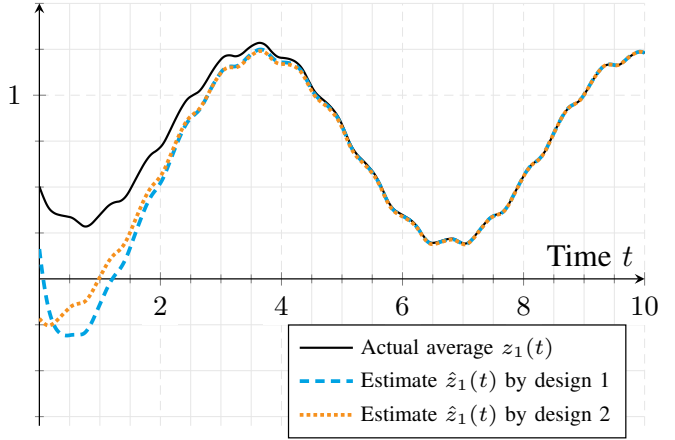

Fig. 2: Average state estimation for Example 1.

Fig. 2 shows the average state estimation by the two design procedures presented in this paper. Note that the state of the observer $\widehat{\Omega}$ is initialized at zero for both designs. The figure, however, shows the first component of the observer's output, which depends on the value of $v_{1}$ that is different for both designs. Therefore, we have different initial estimates.

Design 1. Choose $v_{1}=1$ and $\mathbf{v}_{2}=1$. Compute $L$ by (13). The desired $\operatorname{eig}(M)=\{-0.75,-1,-2,-3\}$, for which we obtain by pole-placement

$$
K_{1}=\left[\begin{array}{lll}
3.3199 & 2.0276 & 3.0276 \\
4.4858 & 1.2582 & 2.2582 \\
2.4849 & 2.6321 & 2.8821 \\
2.4849 & 1.8821 & 3.6321
\end{array}\right]
$$

where $N=I-L H$ and $K=K_{1}+K_{2}$ with $K_{2}=M L$.

Design 2. Choose $\mathbf{v}_{2}=0$ and $v_{1}=-0.0938$ such that $\lambda_{1}\left(v_{1}\right)=\alpha v_{1}-\beta=-0.75$, where $\alpha, \beta$ are given in (18). Choose $\left\{\lambda_{2}, \lambda_{3}, \lambda_{4}\right\}=\{-1,-2,-3\}$. Then, the matrices $L$ and $K_{1}$ are computed by (14) and $M, N$, and $K$ by (5). Obtain $\widehat{\Omega}_{1}$ with $\lambda_{1}=-0.75$ and $\ell_{1}^{T}=[-0.0312,0.2187,-0.0312]$.

Remark VI.1. For a network system to satisfy (7), it is necessary that every unmeasured node is connected to at least one measured (or output) node as shown in Fig. 1(a). Thus this condition in general requires a large number of measured nodes, which is not feasible due to limited number of available sensors. The purpose of the next example is to show that even if (7) doesn't hold, we obtain a satisfactory average state estimate under the conditions of Theorem V.1.

Example VI.2. Consider a reaction-diffusion system over a grid network shown in Fig.1(b). The state at each node satisfies $\dot{x}_{i}(t)=-r_{i} x_{i}(t)+\sum_{j=1}^{n} a_{i j}\left[x_{j}(t)-x_{i}(t)\right]$, where $r_{i}=0.2$ is the reaction rate and $a_{i j}=a_{j i}$ is the diffusion rate, [7]. The term $a_{i j}=1$ if the nodes $i$ and $j$ are connected and 0 otherwise, and the output matrix $C=\left[\begin{array}{ll}0_{4 \times 96} & I_{4}\end{array}\right]$. The inputs are such that $u_{1}(t)=\sin (0.05 t)$ applied at nodes 97 and 98, $u_{2}(t)=\sin (t)$ applied at nodes 99 and 100, and $u_{3}(t)=0.01$ applied at the remaining boundary nodes of the grid (Fig.1(b)). We obtain $\widehat{\Omega}_{1}$ by finding $v_{1}^{*}=-0.0011$ by (19), and $\lambda_{1}\left(v_{1}^{*}\right)=$ -0.0178 . For a nonoptimal case, we choose a faster eigenvalue $\lambda_{1}=-0.5$ which gives $v_{1}=-0.0614$.

With faster eigenvalue, we obtain faster convergence in the transient phase, however, the asymptotic estimation error 


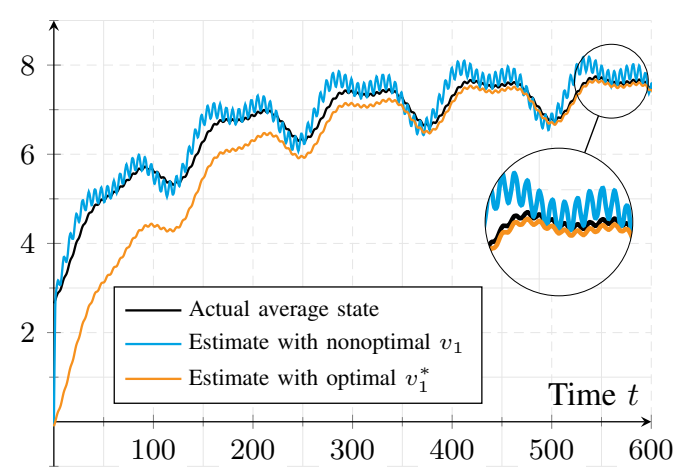

Fig. 3: Average state estimation for Example 2.
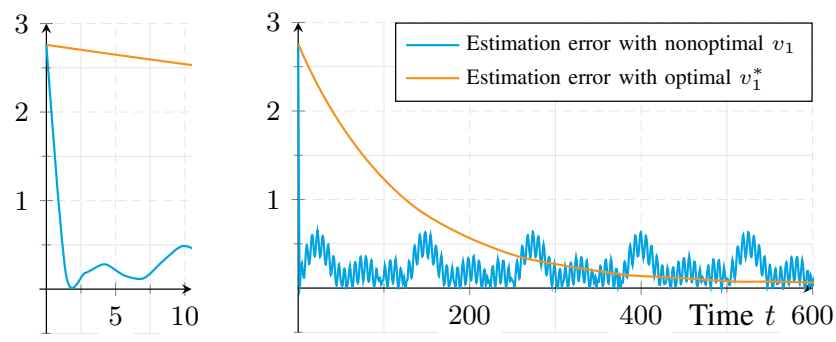

Fig. 4: Estimation error for Example 2.

is larger. The optimal $v_{1}^{*}$ gives slower convergence in the transient phase, however, the asymptotic estimation error is smaller, as shown in Fig. 3 and Fig. 4. This example, therefore, illustrates the minimization setup (18) and the solution (19) that minimizes asymptotic value of the estimation error.

Finally, we observe that even though only 8 unmeasured nodes out of 96 are connected to the measured nodes, which signals a huge distance from the necessary and sufficient condition (7), we are still able to obtain a satisfactory asymptotic estimate of the average state as shown in Fig. 3. This might be the case due to the symmetric structure of the grid, which may have rendered the average deviation vector to have smaller values. However, to present a detailed analysis on this type of behavior is beyond the scope of this paper and is postponed for the future work.

\section{CONCLUSIONS}

We provided a necessary and sufficient condition for the existence of an average state observer and devised two design procedures. Design 1 provides more control over the transient behavior of the observer and design 2 yields an average state observer of dimension one. When the necessary and sufficient condition is not satisfied, an error minimization methodology is devised based on the proposed design procedures. The complexity of the estimation problem and the upper bound on the asymptotic estimation error are shown to not increase with the scale of the system.

The future prospects of this work include an extension to the estimation of averages of multiple clusters or zones in large physical networks. Moreover, estimating nonlinear functionals of the state is also under consideration. For instance, the norm of average deviation vector provides a spread of states around average, which may find applications in output regulation of systems.

\section{ACKNOWLEDGEMENT}

This work is supported by European Research Council (ERC) under the European Union's Horizon 2020 research and innovation programme (ERC-AdG no. 694209, ScaleFreeBack, website: http://scale-freeback.eu/).

\section{REFERENCES}

[1] A. C. Antoulas, Approximation of large-scale dynamical systems. Philadelphia, PA, USA: SIAM, 2005.

[2] S. Coogan and M. Arcak, "A compartmental model for traffic networks and its dynamical behavior," IEEE Transactions on Automatic Control, vol. 60, no. 10, pp. 2698-2703, 2015.

[3] W. Mei, S. Mohagheghi, S. Zampieri, and F. Bullo, "On the dynamics of deterministic epidemic propagation over networks," Annual Reviews in Control, vol. 44, pp. 116-128, 2017.

[4] K. Deng, P. Barooah, P. G. Mehta, and S. P. Meyn, "Building thermal model reduction via aggregation of states," in American Control Conference (ACC), 2010, pp. 5118-5123, 2010.

[5] F. Bullo, Lectures on Network Systems. CreateSpace, 1 ed., 2018. With contributions by J. Cortes, F. Dörfler, and S. Martinez. Link: http://motion.me.ucsb.edu/book-lns.

[6] T. Sadamoto, T. Ishizaki, and J.-I. Imura, "Average state observers for large-scale network systems," IEEE Transactions on Control of Network Systems, vol. 4, no. 4, pp. 761-769, 2017.

[7] T. Ishizaki, K. Kashima, J.-I. Imura, and K. Aihara, "Model reduction and clusterization of large-scale bidirectional networks," IEEE Transactions on Automatic Control, vol. 59, no. 1, pp. 48-63, 2014.

[8] D. Luenberger, "An introduction to observers," IEEE Transactions on Automatic Control, vol. 16, no. 6, pp. 596-602, 1971.

[9] P. Murdoch, "Observer design for a linear functional of the state vector," IEEE Transactions on Automatic Control, vol. 18, no. 3, pp. 308-310, 1973.

[10] M. Aldeen and H. Trinh, "Reduced-order linear functional observer for linear systems," IEE Proceedings-Control Theory and Applications, vol. 146 , no. 5, pp. 399-405, 1999.

[11] M. Darouach, "Existence and design of functional observers for linear systems," IEEE Transactions on Automatic Control, vol. 45, no. 5, pp. 940-943, 2000.

[12] T. L. Fernando, H. M. Trinh, and L. Jennings, "Functional observability and the design of minimum order linear functional observers," IEEE Transactions on Automatic Control, vol. 55, no. 5, pp. 1268-1273, 2010.

[13] M. U. B. Niazi, C. Canudas-de-Wit, and A. Y. Kibangou, "Average observability of large-scale network systems," in European Control Conference (ECC), 2019. hal-02073668.

[14] G. Casadei, C. Canudas-de-Wit, and S. Zampieri, "Controllability of large-scale networks: An output controllability approach," in 2018 IEEE Conference on Decision and Control (CDC), pp. 5886-5891, IEEE, 2018.

[15] P. Kudva, N. Viswanadham, and A. Ramakrishna, "Observers for linear systems with unknown inputs," IEEE Transactions on Automatic Control, vol. 25, no. 1, pp. 113-115, 1980.

[16] M. Darouach, M. Zasadzinski, and S. J. Xu, "Full-order observers for linear systems with unknown inputs," IEEE transactions on Automatic Control, vol. 39, no. 3, pp. 606-609, 1994.

[17] J. Kautsky, N. K. Nichols, and P. Van Dooren, "Robust pole assignment in linear state feedback," International Journal of control, vol. 41, no. 5, pp. $1129-1155,1985$.

[18] S. L. Campbell and C. D. Meyer, Generalized inverses of linear transformations, vol. 56. SIAM, 2009.

[19] R. G. Bartle, The elements of real analysis, vol. 2. Wiley: New York, 1964.

[20] J. K. Merikoski, U. Urpala, A. Virtanen, T.-Y. Tam, and F. Uhlig, "A best upper bound for the 2-norm condition number of a matrix," Linear algebra and its applications, vol. 254, no. 1-3, pp. 355-365, 1997.

[21] G. G. Walter and M. Contreras, Compartmental modeling with networks. Springer-Birkhäuser Boston, 1999. 